\title{
Author Correction: Genetic meta-analysis of diagnosed Alzheimer's disease identifies new risk loci and implicates $A \beta$, tau, immunity and lipid processing
}

Brian W. Kunkle (D), Benjamin Grenier-Boley, Rebecca Sims, Joshua C. Bis, Vincent Damotte, Adam C. Naj, Anne Boland, Maria Vronskaya, Sven J. van der Lee (D), Alexandre Amlie-Wolf(D), Céline Bellenguez (D), Aura Frizatti, Vincent Chouraki, Eden R. Martin, Kristel Sleegers (D), Nandini Badarinarayan, Johanna Jakobsdottir, Kara L. Hamilton-Nelson, Sonia Moreno-Grau, Robert Olaso, Rachel Raybould, Yuning Chen D, Amanda B. Kuzma, Mikko Hiltunen, Taniesha Morgan, Shahzad Ahmad, Badri N. Vardarajan, Jacques Epelbaum, Per Hoffmann, Merce Boada, Gary W. Beecham, Jean-Guillaume Garnier, Denise Harold, Annette L. Fitzpatrick, Otto Valladares, Marie-Laure Moutet, Amy Gerrish, Albert V. Smith, Liming Qu, Delphine Bacq, Nicola Denning, Xueqiu Jian, Yi Zhao, Maria Del Zompo, Nick C. Fox, Seung-Hoan Choi, Ignacio Mateo, Joseph T. Hughes, Hieab H. Adams ID, John Malamon, Florentino Sanchez-Garcia, Yogen Patel, Jennifer A. Brody (D), Beth A. Dombroski, Maria Candida Deniz Naranjo, Makrina Daniilidou, Gudny Eiriksdottir, Shubhabrata Mukherjee, David Wallon, James Uphill, Thor Aspelund (D, Laura B. Cantwell, Fabienne Garzia, Daniela Galimberti, Edith Hofer, Mariusz Butkiewicz, Bertrand Fin, Elio Scarpini, Chloe Sarnowski, Will S. Bush D, Stéphane Meslage, Johannes Kornhuber, Charles C. White, Yuenjoo Song, Robert C. Barber (D), Sebastiaan Engelborghs, Sabrina Sordon, Dina Voijnovic, Perrie M. Adams, Rik Vandenberghe, Manuel Mayhaus, L. Adrienne Cupples (D), Marilyn S. Albert, Peter P. De Deyn (D), Wei Gu, Jayanadra J. Himali (D), Duane Beekly, Alessio Squassina (D), Annette M. Hartmann, Adelina Orellana, Deborah Blacker, Eloy Rodriguez-Rodriguez, Simon Lovestone, Melissa E. Garcia, Rachelle S. Doody, Carmen Munoz-Fernadez, Rebecca Sussams, Honghuang Lin iD, Thomas J. Fairchild, Yolanda A. Benito, Clive Holmes, Hata Karamujić-Čomić, Matthew P. Frosch, Hakan Thonberg, Wolfgang Maier, Gennady Roshchupkin (D), Bernardino Ghetti D, Vilmantas Giedraitis (D), Amit Kawalia, Shuo Li (D), Ryan M. Huebinger, Lena Kilander, Susanne Moebus, Isabel Hernández, M. Ilyas Kamboh, RoseMarie Brundin, James Turton, Qiong Yang (D), Mindy J. Katz, Letizia Concari, Jenny Lord, Alexa S. Beiser, C. Dirk Keene, Seppo Helisalmi, Iwona Kloszewska, Walter A. Kukull, Anne Maria Koivisto, Aoibhinn Lynch, Lluís Tarraga, Eric B. Larson, Annakaisa Haapasalo, Brian Lawlor, Thomas H. Mosley, Richard B. Lipton, Vincenzo Solfrizzi, Michael Gill (D, W. T. Longstreth Jr, Thomas J. Montine, Vincenza Frisardi, Monica Diez-Fairen, Fernando Rivadeneira (D),

Ronald C. Petersen, Vincent Deramecourt, Ignacio Alvarez, Francesca Salani, Antonio Ciaramella, Eric Boerwinkle, Eric M. Reiman, Nathalie Fievet, Jerome I. Rotter, Joan S. Reisch, Olivier Hanon, Chiara Cupidi, A. G. Andre Uitterlinden, Donald R. Royall, Carole Dufouil, Raffaele Giovanni Maletta, Itziar de Rojas, Mary Sano, Alexis Brice, Roberta Cecchetti, Peter St George-Hyslop, Karen Ritchie, Magda Tsolaki D, Debby W. Tsuang, Bruno Dubois, David Craig, Chuang-Kuo Wu, Hilkka Soininen, Despoina Avramidou, Roger L. Albin, Laura Fratiglioni, Antonia Germanou, Liana G. Apostolova, Lina Keller, Maria Koutroumani, Steven E. Arnold, Francesco Panza, Olymbia Gkatzima, Sanjay Asthana, Didier Hannequin, Patrice Whitehead, Craig S. Atwood (D), Paolo Caffarra, Harald Hampel, Inés Quintela, Ángel Carracedo, Lars Lannfelt, David C. Rubinsztein (D, Lisa L. Barnes, Florence Pasquier (D), Lutz Frölich, Sandra Barral, Bernadette McGuinness, Thomas G. Beach, Janet A. Johnston, James T. Becker (D), Peter Passmore, Eileen H. Bigio, Jonathan M. Schott (D), Thomas D. Bird, Jason D. Warren, Bradley F. Boeve, Michelle K. Lupton (D), James D. Bowen (D, Petra Proitsi, Adam Boxer, John F. Powell, James R. Burke (iD, John S. K. Kauwe, Jeffrey M. Burns, Michelangelo Mancuso (D), Joseph D. Buxbaum, Ubaldo Bonuccelli, Nigel J. Cairns, Andrew McQuillin (D), Chuanhai Cao, Gill Livingston, Chris S. Carlson, Nicholas J. Bass, Cynthia M. Carlsson, John Hardy, Regina M. Carney, Jose Bras, Minerva M. Carrasquillo, Rita Guerreiro, Mariet Allen, Helena C. Chui, Elizabeth Fisher D, Carlo Masullo, Elizabeth A. Crocco, Charles DeCarli, Gina Bisceglio, Malcolm Dick, Li Ma, Ranjan Duara, Neill R. Graff-Radford, Denis A. Evans, Angela Hodges, Kelley M. Faber, Martin Scherer, Kenneth B. Fallon, Matthias Riemenschneider, David W. Fardo (D), Reinhard Heun, Martin R. Farlow, Heike Kölsch, Steven Ferris, Markus Leber, Tatiana M. Foroud, Isabella Heuser, Douglas R. Galasko, Ina Giegling, Marla Gearing, Michael Hüll, Daniel H. Geschwind, John R. Gilbert, John Morris, Robert C. Green, Kevin Mayo, John H. Growdon, Thomas Feulner, Ronald L. Hamilton (D), Lindy E. Harrell, Dmitriy Drichel, Lawrence S. Honig, Thomas D. Cushion, Matthew J. Huentelman, Paul Hollingworth,

Christine M. Hulette, Bradley T. Hyman, Rachel Marshall, Gail P. Jarvik, Alun Meggy, Erin Abner, Georgina E. Menzies 1 , Lee-Way Jin, Ganna Leonenko, Luis M. Real, Gyungah R. Jun, Clinton T. Baldwin, Detelina Grozeva, Anna Karydas, Giancarlo Russo, Jeffrey A. Kaye, Ronald Kim, Frank Jessen, Neil W. Kowall, Bruno Vellas, Joel H. Kramer, Emma Vardy, Frank M. LaFerla, Karl-Heinz Jöckel, James J. Lah, Martin Dichgans, James B. Leverenz, David Mann, Allan I. Levey, Stuart Pickering-Brown (D), Andrew P. Lieberman, Norman Klopp, Kathryn L. Lunetta D, H-Erich Wichmann, Constantine G. Lyketsos, Kevin Morgan (D), Daniel C. Marson, Kristelle Brown, Frank Martiniuk, Christopher Medway, 
Deborah C. Mash, Markus M. Nöthen, Eliezer Masliah, Nigel M. Hooper (D, Wayne C. McCormick, Antonio Daniele,

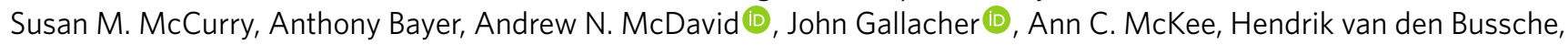
Marsel Mesulam, Carol Brayne, Bruce L. Miller, Steffi Riedel-Heller, Carol A. Miller, Joshua W. Miller, Ammar Al-Chalabi D, John C. Morris, Christopher E. Shaw, Amanda J. Myers, Jens Wiltfang, Sid O'Bryant, John M. Olichney, Victoria Alvarez, Joseph E. Parisi, Andrew B. Singleton, Henry L. Paulson, John Collinge, William R. Perry, Simon Mead, Elaine Peskind, David H. Cribbs, Martin Rossor, Aimee Pierce, Natalie S. Ryan, Wayne W. Poon (1), Benedetta Nacmias, Huntington Potter, Sandro Sorbi, Joseph F. Quinn, Eleonora Sacchinelli(i), Ashok Raj, Gianfranco Spalletta, Murray Raskind, Carlo Caltagirone, Paola Bossù $\mathbb{D}$, Maria Donata Orfei, Barry Reisberg,

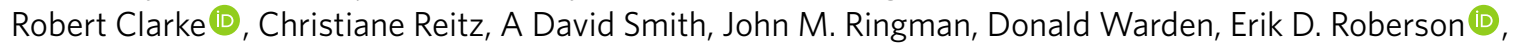
Gordon Wilcock, Ekaterina Rogaeva, Amalia Cecilia Bruni, Howard J. Rosen, Maura Gallo, Roger N. Rosenberg, Yoav Ben-Shlomo, Mark A. Sager, Patrizia Mecocci, Andrew J. Saykin (D), Pau Pastor (D), Michael L. Cuccaro, Jeffery M. Vance, Julie A. Schneider, Lori S. Schneider, Susan Slifer, William W. Seeley, Amanda G. Smith, Joshua A. Sonnen, Salvatore Spina, Robert A. Stern, Russell H. Swerdlow, Mitchell Tang, Rudolph E. Tanzi®D, John Q. Trojanowski, Juan C. Troncoso, Vivianna M. Van Deerlin, Linda J. Van Eldik, Harry V. Vinters, Jean Paul Vonsattel, Sandra Weintraub, Kathleen A. Welsh-Bohmer, Kirk C. Wilhelmsen, Jennifer Williamson, Thomas S. Wingo (D), Randall L. Woltjer, Clinton B. Wright, Chang-En Yu, Lei Yu, Yasaman Saba, Alzheimer Disease Genetics Consortium (ADGC), The European Alzheimer's Disease Initiative (EADI), Cohorts for Heart and Aging Research in Genomic Epidemiology Consortium (CHARGE), Genetic and Environmental Risk in AD/Defining Genetic, Polygenic and Environmental Risk for Alzheimer's Disease Consortium (GERAD/PERADES), Alberto Pilotto, Maria J. Bullido (D, Oliver Peters, Paul K. Crane, David Bennett, Paola Bosco, Eliecer Coto, Virginia Boccardi, Phil L. De Jager (D), Alberto Lleo, Nick Warner, Oscar L. Lopez, Martin Ingelsson, Panagiotis Deloukas (D), Carlos Cruchaga (D), Caroline Graff, Rhian Gwilliam, Myriam Fornage, Alison M. Goate D, Pascual Sanchez-Juan, Patrick G. Kehoe, Najaf Amin, Nilifur Ertekin-Taner, Claudine Berr (D), Stéphanie Debette, Seth Love, Lenore J. Launer, Steven G. Younkin,

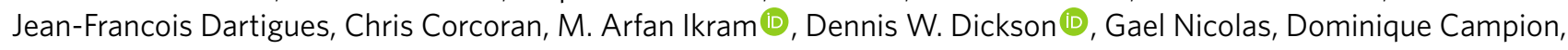
JoAnn Tschanz, Helena Schmidt, Hakon Hakonarson, Jordi Clarimon, Ron Munger, Reinhold Schmidt, Lindsay A. Farrer, Christine Van Broeckhoven (D), Michael C. O’Donovan (D), Anita L. DeStefano, Lesley Jones @ , Jonathan L. Haines, Jean-Francois Deleuze, Michael J. Owen —D, Vilmundur Gudnason, Richard Mayeux, Valentina Escott-Price (D, Bruce M. Psaty, Alfredo Ramirez (1), Li-San Wang, Agustin Ruiz, Cornelia M. van Duijn, Peter A. Holmans, Sudha Seshadri,

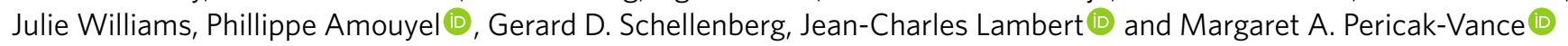

Correction to: Nature Genetics https://doi.org/10.1038/s41588-019-0358-2, published online 28 February 2019.

In the version of this article originally published, the name of author Gennady Roshchupkin was incorrectly spelled as Gena Roschupkin. The error has been corrected in the HTML and PDF versions of the article.

Published online: 15 August 2019

https://doi.org/10.1038/s41588-019-0495-7 